\title{
Falciparum Malaria as an Emerging Cause of Fever in Adults Living in Gabon, Central Africa
}

\author{
Marielle K. Bouyou-Akotet, ${ }^{1,2}$ Christelle L. Offouga, ${ }^{1,2}$ Denise P. Mawili-Mboumba, \\ Laurence Essola, ${ }^{3}$ Blondel Madoungou, ${ }^{3}$ and Maryvonne Kombila ${ }^{1,2}$ \\ ${ }^{1}$ Department of Parasitology Mycology, Faculty of Medicine, Université des Sciences de la Santé, BP 4009 Libreville, Gabon \\ ${ }^{2}$ Malaria Clinical and Operational Research Unit, Regional Hospital of Melen, Libreville, Gabon \\ ${ }^{3}$ Emergency Unit, Centre Hospitalier de Libreville, Libreville, Gabon \\ Correspondence should be addressed to Marielle K. Bouyou-Akotet; mariellebouyou@yahoo.fr
}

Received 18 October 2013; Revised 4 March 2014; Accepted 8 April 2014; Published 30 April 2014

Academic Editor: Seth Owusu-Agyei

Copyright (C) 2014 Marielle K. Bouyou-Akotet et al. This is an open access article distributed under the Creative Commons Attribution License, which permits unrestricted use, distribution, and reproduction in any medium, provided the original work is properly cited.

\begin{abstract}
Following the observed increase of malaria prevalence among older children in Gabon, a descriptive observational study was carried out in 2012 to determine the prevalence of malaria in adults presenting with fever in two health centres of Libreville, the capital city of Gabon. Thick- and thin-blood smears for malaria diagnosis were performed in febrile individuals aged more than 15 years old. Age, use of bed nets, previous antimalarial drug treatment, clinical symptoms, chest radiography results, and available haemoglobin data were also recorded. Among the 304 patients screened, the global malaria frequency was of $42.1 \%(n=128 / 34)$. Plasmodium $(P)$. falciparum was the only species identified. The proportion of patients with a clinical malaria requiring parenteral treatment was $38.5 \%$, whereas $47.5 \%$ of outpatients had uncomplicated malaria. According to WHO classification, 14 (19.7\%) infected patients had severe malaria; neurological and respiratory symptoms tended to be more frequent in case of $P$. falciparum infection. Anaemia was found in 51.5\% adults and none had severe anaemia. Almost half of adults consulting for fever in two health centres of the urban city of Libreville have malaria. The use of insecticide-treated bed nets, the screening, and the treatment of individuals with $P$. falciparum microscopic and submicroscopic asymptomatic infection or clinical malaria should be emphasized to reduce the transmission.
\end{abstract}

\section{Introduction}

In areas with a high level of malaria transmission, children under five years of age and pregnant women represent the most vulnerable population and the primary target of prevention strategies. Reported malaria cases in African adults are scarce. The risk of malaria attacks in residents of malaria-endemic areas decreases with age, because of sequentially acquired immunity which leads to a reduction of life-threatening disease, incidence of mild malaria among adults, and parasite prevalence $[1,2]$. However, recent data suggest that malaria burden may be much more frequent than described in nonpregnant adults [3-5].

After the decline in the proportion of malaria cases in Gabonese children between 2005 and 2008, a rebound in infection rates in rural and urban areas occurred [6]. Malaria risk increased over time among older children and decreased among those aged less than five years who benefitted from free preventive measures and treatment over the last seven years $[6,7]$. Results have also suggested that in Libreville, malaria transmission remains perennial but becomes mesoendemic, with features of urban epidemiology $[6,7]$.

Aside from entomological and immunological surveys, measurements of malariometric indices in the exposed population are also essentials to characterize the transmission. Unrecognised parasite reservoir such as asymptomatic or submicroscopic carriers, along with undiagnosed infected adults can contribute to the sustainment of malaria transmission $[5,8,9]$. In Gabon, most epidemiological studies have focused on children, pregnant women, and asymptomatic adults [6, 10-12]. However, in some African settings, it has been reported that severe clinical attacks also occur in 
adults living in areas with stable malaria and that malaria considerably contributes to death in adults $[13,14]$. The last report of clinical malaria attacks in Gabonese adults was done more than 20 years ago; it indicated a prevalence of $12.6 \%$ [15].

The present study was designed to determine the prevalence of malaria in adults consulting for fever in two hospitals of Gabon, Central Africa, and P. falciparum attacks clinical features at admission.

\section{Patients and Methods}

2.1. Study Site and Population. This is an observational and descriptive study using data collected during a prospective cross-sectional survey conducted from December 2011 to October 2012 in Gabon, at Libreville, the capital city, and at Melen, a suburban area located $11 \mathrm{~km}$ north of Libreville. Patients were recruited at the Centre Hospitalier Universitaire de Libreville (CHL) and the regional hospital of Melen (RHM), two sentinel sites for malaria surveys selected by the Malaria National Control Program (MNCP). In sentinel sites, febrile patients are routinely screened for malaria infection; therefore, data collection was part of the normal activities at the CHL and at the RHM. As malaria transmission is perennial without significant fluctuation throughout the year, the study team collected data over at least one rainy and one dry season in each site.

Febrile outpatients and inpatients, aged more than 15 years, were included after they accepted to participate in the study. Oral informed consent was obtained to complete the demographic and medical history sections of a case report form (CRF). Tympanic temperature, history and duration of fever, age, sex, bed net use, prior self-treatment with an antimalarial drug, and clinical symptoms leading to the consultation were recorded into the CRF. In order to minimize the frequency of coincidental parasitaemia in case of comorbidity, patients with known chronic infection such as HIV, hepatitis, and tuberculosis were not included.

2.2. Malaria Diagnosis. For each patient, matched thickand thin-blood smears were prepared and stained with $20 \%$ Giemsa. Thick smears were screened for the presence of malaria parasites according to the Lambaréné method [16]. Carefully, $10 \mu \mathrm{L}$ of blood was layed on a 10 by $18 \mathrm{~mm}$ area of a microscope slide and then dried and stained. Parasitemia was expressed as the number of parasites per microlitre of blood $(\mathrm{p} / \mu \mathrm{L})$, and parasite species were identified in the matched thin-blood smears. Smears were read using a light microscope ( $\times 100$ oil immersion lenses). Quality control of the blood smears was performed: a second microscopist, blinded to the results of the first reading, read the slides. In case of disagreement, slides were controlled by a third microscopist and the mean of the two closest readings of parasitaemia was taken. A patient was considered negative if no parasite was observed after the examination of at least 100 oil immersion fields in a thick-blood smear.

2.3. Haematology. Available haemoglobin ( $\mathrm{Hb})$ measurements were used for the analysis of anaemia. According to the WHO classification, anaemia was defined as a $\mathrm{Hb}$ concentration below $11 \mathrm{~g} / \mathrm{dL}$ and classified as severe $(\mathrm{Hb}<$ $5 \mathrm{~g} / \mathrm{dL})$, moderate $(5 \geq \mathrm{Hb}<8 \mathrm{~g} / \mathrm{dL})$, or low $(8 \geq \mathrm{Hb}<11 \mathrm{~g} / \mathrm{dL})$ type.

2.4. Definitions. According to WHO 2010 and Newton and Krishna classification of African population modes of disease presentation, malaria patients were classified into three groups: (1) severe malaria patients who strictly met the WHO 2000 criteria for severe malaria; (2) moderate malaria patients who did not have any clinical or biological features of SM according to WHO classification but required parenteral treatment and hospitalization because of symptoms such as asthenia, vomiting, convulsions; (3) uncomplicated malaria patients, that were, febrile outpatients who did not have any features of severe or moderate disease and where able to tolerate oral medication $[17,18]$.

Fever was defined as a tympanic temperature $>37.5^{\circ} \mathrm{C}$, dyspnoea as an observed respiratory rate $\geq 20$ beats/minutes. Level of consciousness was determined using the Glasgow coma score and impaired consciousness as a GCS $<10$. Any degree of impairment of consciousness or any other neurological involvement was considered as a potentially serious neurological manifestation. Chest radiography results were used for the diagnosis of pulmonary infection.

2.5. Ethical Consideration. The study aims were clearly explained to the ward staffs. Patients were informed about the protocol, and their oral consent was required prior to data collection. Although it was a noninvasive study, this oral consent was sought to obtain medical history and to use the demographic, clinical and biological data. Data were kept confidential. The study was approved by the Ministry of Health. As a reference laboratory for the MNCP, the Department of Parasitology Mycology (DPM) has health authorities' approval to monitor the evolution of malaria morbidity throughout the entire country through sentinel sites. Malaria diagnosis was free of charge and infected patients were treated according to the national policy. For patients aged between 15 and 20 years old, individual oral assent and oral consent of legal guardian were required prior to their inclusion.

2.6. Sample Size Calculation. According to WHO sample size tables for prevalence studies, the sample size calculation was based on 5\% precision with $95 \%$ level of confidence and an expected prevalence of symptomatic malaria of $15 \%$ [19]. This expected prevalence was based on the assumption that the prevalence of symptomatic adults will be at least comparable to the reported prevalence of asymptomatic malaria in the country $[11,12]$. Therefore a minimum of 196 screened adults was necessary.

2.7. Statistical Analysis. All data were entered and cleaned using Epi-info version 3.3.2 (2005 CDC Atlanta) and analyzed with Stata. Qualitative variables were summarised as frequencies and percentages. Median with interquartile ranges (percentiles 25 th and 75 th) were used for continuous 
TABLE 1: Patient characteristics.

\begin{tabular}{|c|c|c|c|c|}
\hline Continuous variables & All $(N=304)$ & Malaria-positive $(n=128)$ & $\begin{array}{c}\text { Uninfected } \\
(n=176)\end{array}$ & $P$ \\
\hline Age, years* & $25(19-34)$ & $24(19-33)$ & $26(20-2)$ & 0.37 \\
\hline Duration of illness, days* & $2(2-4)$ & $2(2-5)$ & $2(2-2)$ & 0.88 \\
\hline Haemoglobin, $\mathrm{g} / \mathrm{dL}^{*}$ & $10.8(10.1-11.8)$ & $11.0(9.9-11.7)$ & $10.8(10.1-11.8)$ & 0.14 \\
\hline Outpatients, $n(\%)$ & $120(39.5)$ & $57(44.5)$ & $63(35.8)$ & 0.12 \\
\hline Inpatients, $n(\%)$ & $184(60.5)$ & $71(55.5)$ & $113(64.2)$ & 0.12 \\
\hline Bed net use, $n(\%)$ & $53(17.4)$ & $23(17.90)$ & $30(17.1)$ & 0.83 \\
\hline Previous self-treatment with antimalarial, $n(\%)^{* *}$ & $30(15.3)$ & $9(12.7)$ & $21(16.8)$ & 0.27 \\
\hline Anaemia, $n(\%)^{* * *}$ & $105(51.5)$ & $39(54.5)$ & $66(47.0)$ & 0.19 \\
\hline
\end{tabular}

${ }^{*}$ Median (IQR). ${ }^{* *}$ Data available for 196 patients. ${ }^{* * *}$ Data available for 204 patients.

variables such as age, $\mathrm{Hb}$, and parasitaemia. The results were compared with the Chi-square or Fisher's exact test or the nonparametric tests (Mann Whitney and Kruskal Wallis) as appropriate. Bivariate analysis was used to determine possible factors that could be associated with malaria. A multivariate analysis was then performed using a forward stepwise likelihood ratio logistic regression, and considering the presence of malaria parasite (Yes/No) as the dependent variable, and variables which resulted in a $P$ value of $<0.20$ in the bivariate analysis as the independent variables for this analysis. The significance level of all comparisons was set at a $P$ value $<0.05$.

\section{Results}

During the study period, 1654 febrile patients were screened for malaria by the study team, only 361 (22\%) were adults. Data from 304 were available and analysed. One hundred twenty patients were referred by the outpatient unit, and 184 came from the emergency ward. The median age of the patients was 25 [19-34] years, and $29 \%(n=88)$ were male. The majority of the patients attended the hospital after two days of illness duration (2 [2-5] days), and $15.3 \%(n=30 / 196)$ of them had taken an antimalarial treatment before the consultation (Table 1). Artemisinin-based combination therapies (ACTs) represented $99 \%$ of the used drugs for previous selfmedication; only one patient had taken tablets of quinine before the consultation. The median temperature was 39.0 $[38.5-39.0]^{\circ} \mathrm{C}$. Haemoglobin values that were available for 204 patients, ranged between $5.6 \mathrm{~g} / \mathrm{dL}$ and $15.3 \mathrm{~g} / \mathrm{dL}$. Anaemia was detected in more than half of them and $18(8.8 \%)$ had moderate anaemia. Bed net use was infrequent (Table 1).

3.1. Malaria Infection. A total of $128(42.1 \%)$ among the 304 adults had a positive blood smear, with only three harbouring gametocytes with asexual forms. P. falciparum was the only species identified. Parasite densities varied from 28 to $214775 \mathrm{p} / \mu \mathrm{L}$, the median value being 5600 [869-23310] p/ $\mu \mathrm{L}$. Thirty-six (28.1\%) out of the 128 infected patients had a parasite count below $1000 \mathrm{p} / \mu \mathrm{L}, 61(47.7 \%)$ had more than $5,000 \mathrm{p} / \mu \mathrm{L}$, and five $(3.9 \%)$ had a count greater than $100,000 \mathrm{p} / \mu \mathrm{L}$. The $P$. falciparum infection rate did not vary significantly with bed net use or previous antimalarial treatment (Table 1). Malaria-positive and uninfected groups were almost indistinguishable in terms of the time between the onset of symptoms and presentation at hospital, with a median of two days. Infected patients reported maximum fever duration of six days, whereas fever was present up to a maximum of 23 days before consultation in the group of malaria-negative patients. The proportion of patients with malaria infection requiring parenteral treatment (moderate or severe malaria) was $38.6 \%$ ( $n=71 / 184$ inpatients), whereas $47.5 \%$ ( $n=57 / 120$ outpatients) had uncomplicated malaria $(P=0.1)$. $P$. falciparum prevalence did not correlate with age: it was $44.8 \%$ in patients aged less than 31 years, $34.6 \%$ among those age between 31 and 45 years, and $43.5 \%$ in those over 45 years.

3.2. Symptoms Associated with Malaria. Among the malaria patients, $77.3 \%(n=99 / 128)$ did not present clinical and radiological signs suggestive of other infection. For outpatients, the main reasons for consultation, except fever or history of fever, were fatigue and headache. In the hospitalized group, fatigue, digestive symptoms, pallor, and respiratory symptoms (more than $25 \%$ of patients) predominated (Table 2). Aside from fever, a total of 26 (36.6\%) of them had multiple symptoms; the predominant association was represented by the vomiting plus pallor and fatigue (21.1\%). The majority of the infected inpatients $(n=57 / 71)$ had moderate malaria. Clinical signs of severe malaria were present in 14 (19.7\%) $P$. falciparum infected inpatients: six (42.9\%) had neurological forms (OR 5.1 95\%CI 1.0-26.1), three (21.4\%) had respiratory distress syndrome, and five (35.7\%) had repeated vomiting (Table 2).

After the multivariate analysis, none of the recorded clinical symptoms was significantly associated with malaria (Table 2).

3.3. Anaemia. Haemoglobin levels were analysed in the subgroup of 204 patients with available data. The majority of anaemic patients ( $n=87 / 105 ; 82.9 \%)$ had low anaemia. $\mathrm{Hb}$ levels and anaemia prevalence were not related to the presence of malaria parasites: 44 among 83 (53.0\%) infected patients and 55 out of $121(45.4 \%)$ had a Hb level below $11 \mathrm{~g} / \mathrm{dL}$. No case of severe malarial anaemia (Hb below $5 \mathrm{~g} / \mathrm{dL}$ with positive blood smear) was observed. Five malaria patients had 


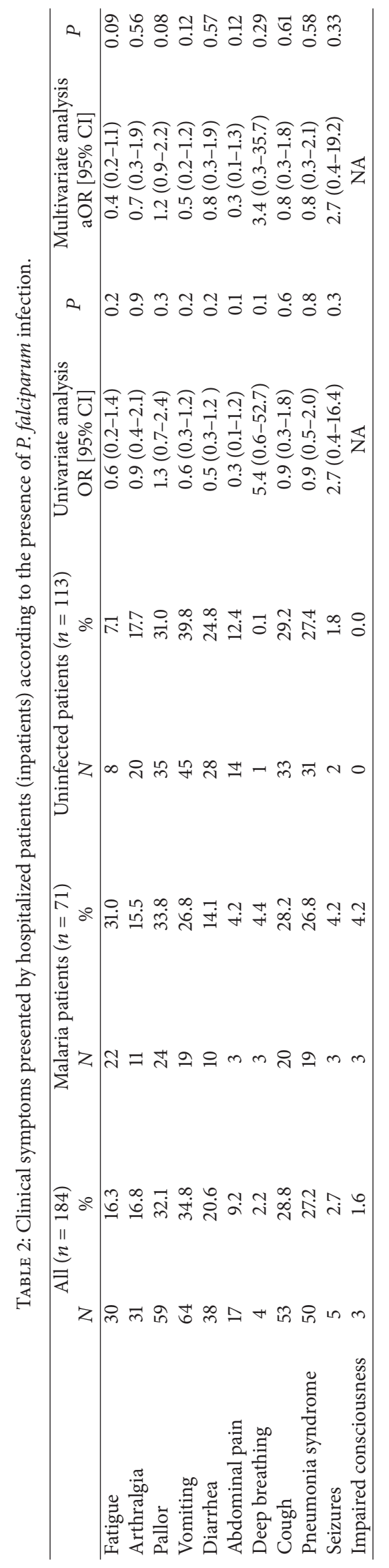


a $\mathrm{Hb}$ level below $7.0 \mathrm{~g} / \mathrm{dL}$, whereas the lowest $\mathrm{Hb}$ value was $7.1 \mathrm{~g} / \mathrm{dL}$ among the uninfected patients.

\section{Discussion}

Since the adoption and implementation of ACTs and insecticide-treated nets (ITNs), efforts have been made to obtain data on population-based prevalence and transmission of malaria in several malaria-endemic countries. These informations should help to identify priority areas for evaluation, monitoring, and improvement of implemented disease control strategies. Epidemiological studies generally focus on children, pregnant women, and asymptomatic individuals $[7,10,20,21]$. However, following the decrease in global malaria prevalence observed over the past few years, the WHO recommends universal ITN coverage to further reduce malaria transmission. Recent data on the burden of malaria in adults from areas with perennial transmission are lacking. These adults are considered to be semi-immune and would represent an important parasite reservoir because they are frequently asymptomatic or submicroscopic parasite carriers $[9,22,23]$. Moreover, the epidemiology of $P$. falciparum is changing through urbanization and implementation of interventions $[24,25]$. This decline could have an impact on the level of acquired specific immunity; thus, the clinical and epidemiological features of malaria infection could be modified according to age $[26,27]$.

Compared to the reported prevalence among children aged less than five years old (17.9\%), a surprisingly high microscopic prevalence of malaria was found among febrile adults living in the capital city of Gabon, Central Africa [6]. This prevalence is threefold higher than those reported 20 years ago (42.1\% versus $19.0 \%)$ and three- to seven fold higher than that of asymptomatic adults living in different areas of Gabon [11, 12, 15]. Recent reports have already highlighted the greatest frequency of malaria in febrile older children in different areas of the country $[6,7]$. A persistent high number of malaria cases in older children and in adults were observed in Zimbabwe, following the scale up of disease control strategies [5]. Therefore, the population who did not benefit for free malaria interventions (i.e., children older than 5 years old and nonpregnant adults) now carries the highest burden of infection. Our results support the notion of a changing malaria epidemiology profile in Libreville. Indeed, changes in malaria transmission are preceded with a shift of malaria burden according to age [21]. The epidemiology of malaria is also modified through urbanisation and implementation of interventions [26-28]. For example, in Thies, an urban area of Senegal with seasonal mesoendemic malaria, the most affected population was aged 4 to 20 years [29]. Libreville is actually undergoing unplanned urbanisation, which connotes proximity to mosquito breeding grounds. In Uganda, it was reported that malaria risk in adults is associated with this proximity and with the absence of bed net use [30].

A low frequency of parasite density (PD) below $1000 \mathrm{p} / \mu \mathrm{L}$ was also noticed. The median PD was $5600 \mathrm{p} / \mu \mathrm{L}$, higher than the mean PD observed in adult hospitalized patients at the
CHL in $1991(1000 \mathrm{p} / \mu \mathrm{L})$ and the PD in asymptomatic patients from Lambaréné $(<200 \mathrm{p} / \mu \mathrm{L})[11,15]$.

Identifying clinical factors associated with malaria can help to conclude to an incidental parasitaemia or to provide appropriate management of febrile infected patients, at least to identify those who require intensive care unit admission. Gabonese children with complicated malaria present more frequently prostration, seizures, and pallor; the predominant signs, presented by the parasitaemic hospitalized adults, were also found in a similar frequency among uninfected individuals [31]. Altered level of consciousness and seizures were the main neurological symptoms in the case of a positive blood smear as reported in Tanzanian adults [32]. The observed absence of significant clinical risk factors could be explained by a coincidental $P$. falciparum in patients with a fever due to other infections. However, it was recently reported that intensifying malaria control modifies the threshold of parasitaemia to be used to define $P$. falciparum attacks, that is, fever attributable to malaria; pyrogenic threshold levels of $1000 \mathrm{p} / \mu \mathrm{L}$ for adults and of $5000 \mathrm{p} / \mu \mathrm{L}$ in children for attributing fever episodes to $P$. falciparum malaria were defined in an endemic area of Senegal [26]. Thus, the high prevalence and the high parasite densities observed here indicate malaria as the leading cause of the fever, although clinical symptoms of patients remain unspecific. Therefore, there is actually a substantial proportion of febrile adults (38\%) who had a malaria attack classified as either moderate or severe according to WHO and Newton and Krishna classifications $[17,18]$. Nineteen percent of hospitalized patients had WHOdefined severe falciparum malaria. A higher frequency was reported in adults from Asia and non-malaria-endemic settings $[33,34]$. Studies performed the last 20 years confirm the lowest prevalence of SM in African population who already experienced malaria episodes [33-35].

Although this was not a study designed to analyze the risk factors for severe malaria in adults, most of the clinical symptoms presented by the patients were not found to be associated with the presence of $P$. falciparum like recently reported by Newton et al. [36]. However, neurological symptoms and respiratory distress that are recognized as predictive of deaths in adults were also more prevalent in case of positive blood smear [36, 37].

More than half (51.5\%) of the patients had anaemia, which was not significantly related to the presence of malaria parasites. The majority of anaemic individuals had low anaemia like that reported in other settings [34]. According to WHO, a severe public health problem is a disease with a prevalence greater than $40 \%$ in any group. Therefore, evaluating the real burden of anaemia in adult Gabonese population is needed to determine strategies for management and prevention after eliminating known risk factors such as HIV infection, tuberculosis, and arboviruses which are also frequent in adults malaria patients $[38,39]$. Nutritional deficiencies and malaria are the main contributors of low Hb levels in African children; further studies on adults are required. Additionally, submicroscopic parasitaemia and multiplicity of infections that are known to be associated with anaemia were also often observed in adults living in Libreville [40, 41]. 
This study has some limitations. First, it is health-facilitybased study and it did not include patients within the community. It is important to underline that, in the case of fever, a majority of people aged more than 15 years directly obtain an antimalarial drug from a pharmacist; also some patients could have been missed by the study team although they had a daily presence at hospital. Therefore, the burden of malaria could be overestimated or underestimated. A second limitation could be the absence of molecular diagnosis of malaria, which is essential for the real estimation of malaria burden [8].

\section{Conclusion}

This descriptive study highlights the emergence of malaria in febrile adults living in an urban city of Gabon. Malaria attacks which require parenteral treatment are now more frequently described in adult patients, with a prevalence of severe malaria exceeding $10 \%$. Whether this increased prevalence is due to high exposure or to an inadequate acquired immunity remains an important issue. Nevertheless, this population may contribute to the recent rebound of malaria observed in the country as an important parasite reservoir. Additionally, the present data highlight a challenge to control and eliminating efforts in the country. Adults with any evidence of $P$. falciparum infection should be promptly treated with antimalarials before further investigation on other possible causes. The use of ITNs, screening and treatment of individuals with microscopic or submicroscopic asymptomatic infection or clinical malaria should be emphasized and could reduce the transmission of malaria in the country.

\section{Conflict of Interests}

The authors declare no conflict of interests.

\section{Acknowledgments}

The authors are grateful to the patients, to the medical staff of the Emergency Unit of the CHL and the RHM, and to the technical staff of the Department of Parasitology.

\section{References}

[1] M. J. Miller, "Observations on the natural history of malaria in the semi-resistant West African," Transactions of the Royal Society of Tropical Medicine and Hygiene, vol. 52, no. 2, pp. 152$168,1958$.

[2] J.-F. Trape, C. Rogier, L. Konate et al., "The Dielmo project: A Longitudinal Study of natural malaria infection and the mechanisms of protective immunity in a community living in a holoendemic area of Senegal," American Journal of Tropical Medicine and Hygiene, vol. 51, no. 2, pp. 123-137, 1994.

[3] R. Lozano, M. Naghavi, K. Foreman et al., "Global and regional mortality from 235 causes of death for 20 age groups in 1990 and 2010: a systematic analysis for the Global Burden of Disease Study 2010," The Lancet, vol. 380, no. 9859, pp. 2095-2128, 2012.
[4] C. J. L. Murray, L. C. Rosenfeld, S. S. Lim et al., "Global malaria mortality between 1980 and 2010: a systematic analysis," The Lancet, vol. 379, no. 9814, pp. 413-431, 2012.

[5] S. Mharakurwa, S. L. Mutambu, J. Mberikunashe et al., "Changes in the burden of malaria following scale up of malaria control interventions in Mutasa District, Zimbabwe," Malaria Journal, vol. 12, article 223, 2013.

[6] D. P. Mawili-Mboumba, M. K. Bouyou Akotet, E. Kendjo et al., "Increase in malaria prevalence and age of at risk population in different areas of Gabon," Malaria Journal, vol. 12, article 3, 2013.

[7] M. K. Bouyou-Akotet, S. Nzenze-Afène, D. P. MawiliMboumba, M. Owono-Medang, V. Guiyedi, and M. Kombila, "Evolution de la prévalence du paludisme et de l'anémie chez les parturientes à Libreville entre 1995 et 2011," Sante, vol. 21, no. 4, pp. 199-203, 2011.

[8] L. C. Okell, T. Bousema, J. T. Griffin, A. L. Ouédraogo, A. C. Ghani, and C. J. Drakeley, "Factors determining the occurrence of submicroscopic malaria infections and their relevance for control," Nature Communications, vol. 3, p. 1237, 2012.

[9] A. Manjurano, L. Okell, T. Lukindo et al., "Association of sub-microscopic malaria parasite carriage with transmission intensity in north-eastern Tanzania," Malaria Journal, vol. 10, article 370, 2011.

[10] M. K. Bouyou-Akotet, D. P. Mawili-Mboumba, E. Kendjo et al., "Evidence of decline of malaria in the general hospital of Libreville, Gabon from 2000 to 2008," Malaria Journal, vol. 8, no. 1 , article 300, 2009.

[11] M. P. Dal-Bianco, K. B. Köster, U. D. Kombila et al., "High prevalence of asymptomatic Plasmodium falciparum infection in Gabonese adults," American Journal of Tropical Medicine and Hygiene, vol. 77, no. 5, pp. 939-942, 2007.

[12] D. Nkoghe, J.-P. Akue, J.-P. Gonzalez, and E. M. Leroy, "Prevalence of Plasmodium falciparum infection in asymptomatic rural Gabonese populations," Malaria Journal, vol. 10, article 33, 2011.

[13] A. Sowunmi, O. Walker, and L. A. Salako, "Cerebral malaria in non-paediatric subjects resident in southwestern Nigeria," African Journal of Medicine and Medical Sciences, vol. 22, no. 1, pp. 49-53, 1993.

[14] D. Chandramohan, G. H. Maude, L. C. Rodrigues, and R. J. Hayes, "Verbal autopsies for adult deaths: their development and validation in a multicentre study," Tropical Medicine and International Health, vol. 3, no. 6, pp. 436-446, 1998.

[15] J. L. Perret, T. H. Duong, M. Kombila, M. Owono, and C. Nguemby-Mbina, "Results of a systematic search for Plasmodium in internal medicine in Gabon," Bulletin de la Societe de Pathologie Exotique, vol. 84, no. 4, pp. 323-329, 1991.

[16] T. Planche, S. Krishna, M. Kombila et al., "Comparison of methods for the rapid laboratory assessment of children with malaria," American Journal of Tropical Medicine and Hygiene, vol. 65, no. 5, pp. 599-602, 2001.

[17] C. R. J. C. Newton and S. Krishna, "Severe falciparum malaria in children: current understanding of pathophysiology and supportive treatment," Pharmacology and Therapeutics, vol. 79, no. 1, pp. 1-53, 1998.

[18] World Health Organization, "Guidelines for treatment of malaria," 2nd edition, 2010, http://whqlibdoc.who.int/publications/2010/9789241547925_eng.pdf available at http://www.who .int/malaria/en/. 
[19] S. K. Lwanga and S. Lemeshow, Sample Size Determination, World Health Organ, 1991.

[20] E. A. Okiro, A. Al-Taiar, H. Reyburn, R. Idro, J. A. Berkley, and R. W. Snow, "Age patterns of severe paediatric malaria and their relationship to Plasmodium falciparum transmission intensity," Malaria Journal, vol. 8, no. 1, article 4, 2009.

[21] W. P. O’Meara, T. W. Mwangi, T. N. Williams, F. E. McKenzie, R. W. Snow, and K. Marsh, "Relationship between exposure, clinical malaria, and age in an area of changing transmission intensity," American Journal of Tropical Medicine and Hygiene, vol. 79, no. 2, pp. 185-191, 2008.

[22] A. L. Ouédraogo, T. Bousema, S. J. De Vlas et al., “The plasticity of Plasmodium falciparum gametocytaemia in relation to age in Burkina Faso," Malaria Journal, vol. 9, no. 1, article 281, 2010.

[23] A. Ross, G. Killeen, and T. Smith, "Relationships between host infectivity to mosquitoes and asexual parasite density in Plasmodium falciparum," The American Journal of Tropical Medicine and Hygiene, vol. 75, no. 2, pp. 32-37, 2006.

[24] N. Maire, T. Smith, A. Ross, S. Owusu-Agyei, K. Dietz, and L. Molineaux, "A model for natural immunity to asexual blood stages of Plasmodium falciparum malaria in endemic areas," The American Journal of Tropical Medicine and Hygiene, vol. 75, no. 2, pp. 19-31, 2006.

[25] S. J. Ceesay, C. Casals-Pascual, J. Erskine et al., "Changes in malaria indices between 1999 and 2007 in The Gambia: a retrospective analysis," The Lancet, vol. 372, no. 9649, pp. 15451554, 2008.

[26] C. Roucher, C. Rogier, F. Dieye-Ba, C. Sokhna, A. Tall, and J. F. Trape, "Changing malaria epidemiology and diagnostic criteria for Plasmodium falciparum clinical malaria," PLoS One, vol. 7, no. 9, article e46188, 2012.

[27] J.-F. Trape, A. Tall, N. Diagne et al., "Malaria morbidity and pyrethroid resistance after the introduction of insecticidetreated bednets and artemisinin-based combination therapies: A Longitudinal Study," The Lancet Infectious Diseases, vol. 11, no. 12, pp. 925-932, 2011.

[28] M. Caldas De Castro, Y. Yamagata, D. Mtasiwa et al., "Integrated urban malaria control: A Case Study in Dar es Salaam, Tanzania," American Journal of Tropical Medicine and Hygiene, vol. 71, no. 2, pp. 103-117, 2004.

[29] S. J. Ceesay, K. A. Bojang, D. Nwakanma et al., "Sahel, savana, riverine and urban malaria in West Africa: Similar control policies with different outcomes," Acta Tropica, vol. 121, no. 3, pp. 166-174, 2012.

[30] R. L. Pullan, H. Bukirwa, S. G. Staedke, R. W. Snow, and S. Brooker, "Plasmodium infection and its risk factors in eastern Uganda," Malaria Journal, vol. 9, no. 1, article 2, 2010.

[31] M. K. Bouyou-Akotet, D. P. Mawili-Mboumba, E. Kendjo et al., "Complicated malaria and other severe febrile illness in a pediatric ward in Libreville, Gabon," BMC Infectious Diseases, vol. 12, pp. 216-224, 2012.

[32] J. Makani, W. Matuja, E. Liyombo, R. W. Snow, K. Marsh, and D. A. Warrell, "Admission diagnosis of cerebral malaria in adults in an endemic area of Tanzania: implications and clinical description," QJM-Monthly Journal of the Association of Physicians, vol. 96, no. 5, pp. 355-362, 2003.

[33] A. Phillips, P. Bassett, S. Zeki, S. Newman, and G. Pasvol, "Risk factors for severe disease in adults with falciparum malaria," Clinical Infectious Diseases, vol. 48, no. 7, pp. 871-878, 2009.
[34] P. Sagaki, V. Thanachartwet, V. Desakorn et al., "Clinical factors for severity of Plasmodium falciparum Malaria in hospitalized adults in Thailand," PLoS One, vol. 8, article e71503, 2013.

[35] A. Obengui Mbika-Cardorelle, A. Gatshe, P. Talani, and G. Moyen, "Gestion des cas de paludisme de l'adulte en zone d'endémie," Médecine d'Afrique Noire, vol. 51, no. 3, pp. 178-180, 2004.

[36] P. N. Newton, K. Stepniewska, A. Dondorp et al., "Prognostic indicators in adults hospitalized with falciparum malaria in Western Thailand," Malaria Journal, vol. 12, article 229, 2013.

[37] J. Hanson, S. J. Lee, S. Mohanty et al., "Rapid clinical assessment to facilitate the triage of adults with falciparum malaria, a retrospective analysis," PLoS One, vol. 9, no. 1, article e87020, 2014.

[38] I. C. Hendriksen, J. Ferro, P. Montoya et al., "Diagnosis, clinical presentation, and in-hospital mortality of severe malaria in HIV-coinfected children and adults in Mozambique," Clinical Infectious Diseases, vol. 55, no. 8, pp. 1144-1153, 2012.

[39] D. Nkoghe, R. F. Kassa, U. Bisvigou, M. Caron, G. Grard, and E. M. Lero, "No clinical or biological difference between Chikungunya and Dengue Fever during the 2010 Gabonese outbreak," Infectious Disease Reports, vol. 4, no. 1, article e5, 2012.

[40] A. Mayor, J. J. Aponte, C. Fogg et al., "The epidemiology of malaria in adults in a rural area of southern Mozambique," Malaria Journal, vol. 6, article 3, 2007.

[41] R. Nikiéma, D. P. Ndong Ella, M. L. Mawili-Mboumba et al., "Submicroscopic Plasmodium falciparum sexual and asexual carriage in urban and rural areas of Gabon," submitted. 


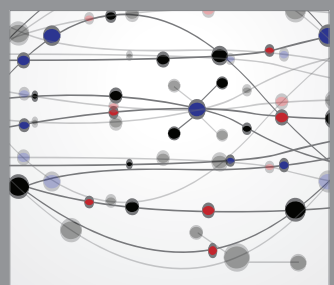

The Scientific World Journal
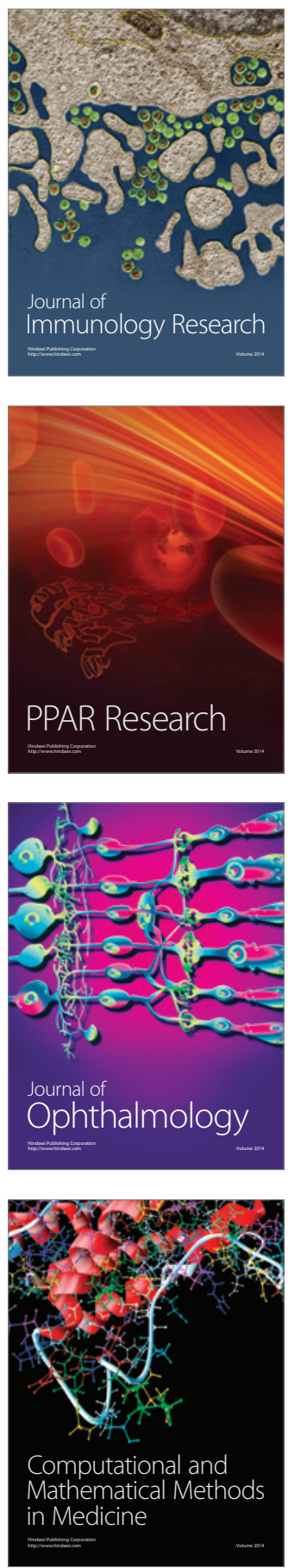

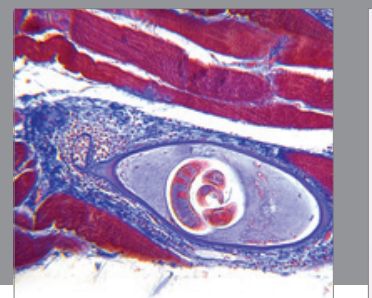

Gastroenterology

Research and Practice
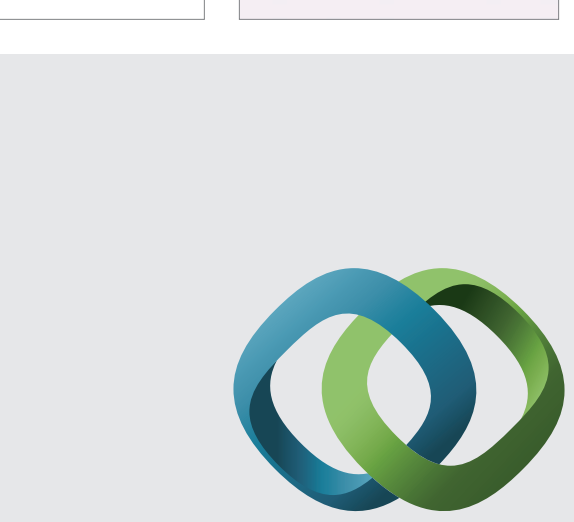

\section{Hindawi}

Submit your manuscripts at

http://www.hindawi.com
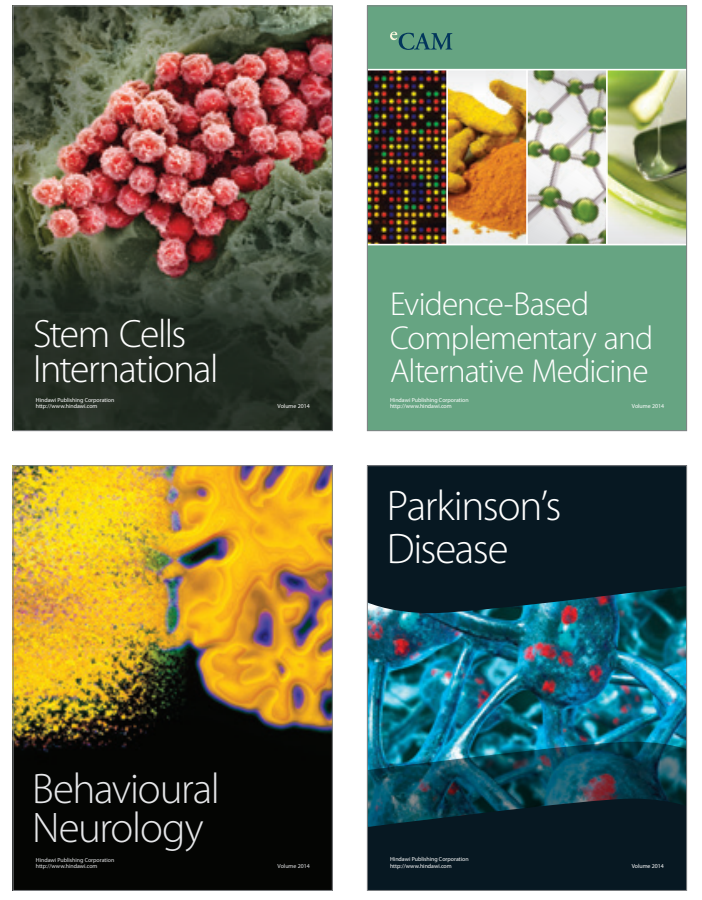
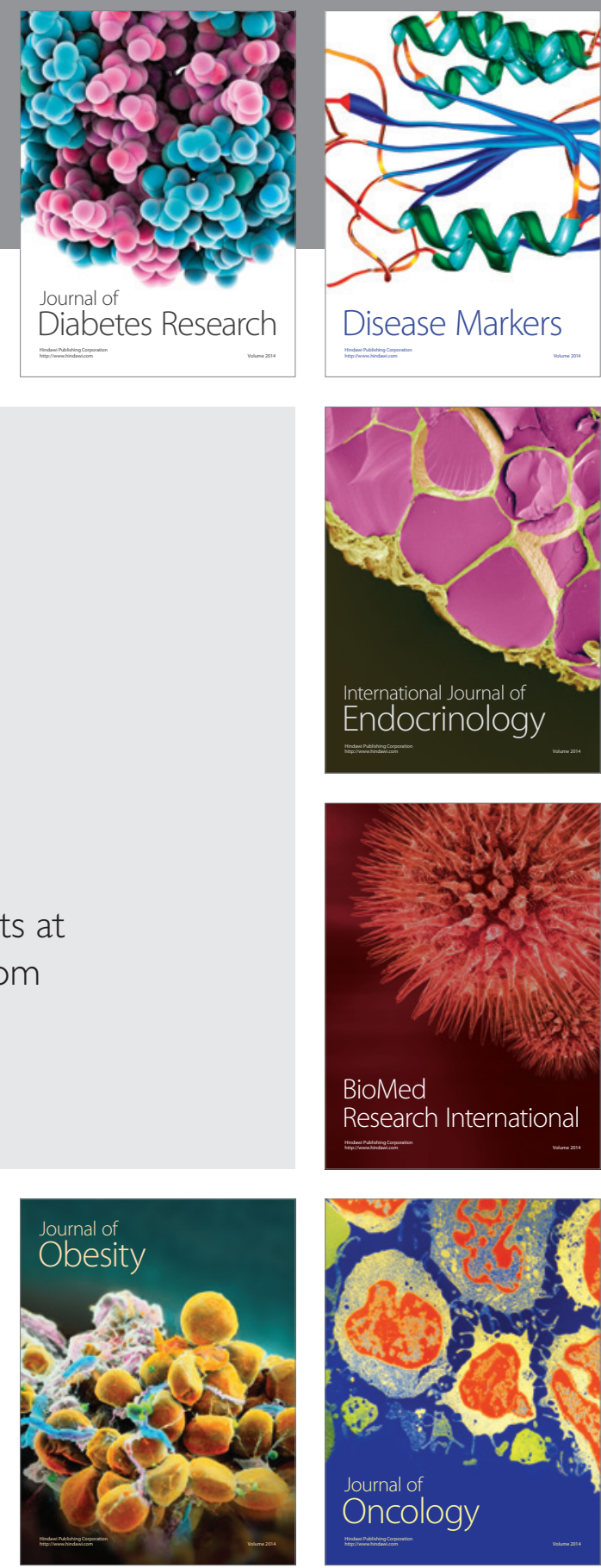

Disease Markers
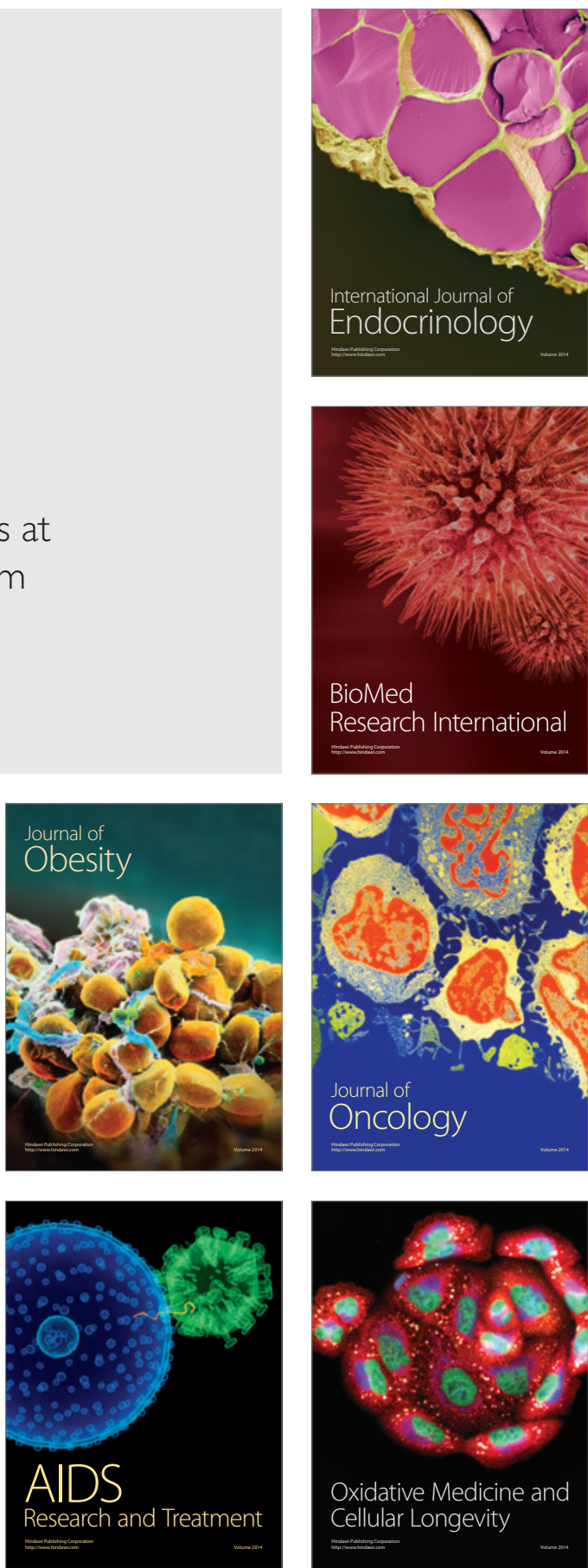